\title{
Related-Key Rectangle Attack on the Full SHACAL-1
}

\author{
Orr Dunkelman ${ }^{1, \star}$, Nathan Keller ${ }^{2, \star \star}$, and Jongsung $\mathrm{Kim}^{3,4, \star \star \star}$ \\ ${ }^{1}$ Computer Science Department, Technion. \\ Haifa 32000, Israel \\ orrd@cs.technion.ac.il \\ ${ }^{2}$ Einstein Institute of Mathematics, Hebrew University. \\ Jerusalem 91904, Israel \\ nkeller@math.huji.ac.il \\ ${ }^{3}$ ESAT/SCD-COSIC, Katholieke Universiteit Leuven \\ Kasteelpark Arenberg 10, B-3001 Leuven-Heverlee, Belgium \\ Kim. Jongsung@esat . kuleuven . be \\ ${ }^{4}$ Center for Information Security Technologies(CIST), Korea University \\ Anam Dong, Sungbuk Gu, Seoul, Korea \\ joshep@cist.korea.ac.kr
}

\begin{abstract}
SHACAL-1 is a 160-bit block cipher with variable key length of up to 512-bit key based on the hash function SHA-1. It was submitted to the NESSIE project and was accepted as a finalist for the 2nd phase of the evaluation.

In this paper we devise the first known attack on the full 80-round SHACAL-1 faster than exhaustive key search. The related-key differentials used in the attack are based on transformation of the collisionproducing differentials of SHA-1 presented by Wang et al.
\end{abstract}

\section{Introduction}

In 1993, NIST has issued a standard hash function called Secure Hash Algorithm (FIPS-180) 25]. Later this version was named SHA-0, as NIST published a small tweak to this standard called SHA-1 in 1995. Both SHA-0 and SHA-1 are based on padding the message and dividing it to blocks of 512 bits, and then iteratively compressing those blocks into a 160-bit digest. Recently, NIST has published three more standard hash functions as part of FIPS-180: SHA-256, SHA-384 and SHA-512. Each of the new hash functions has a digest size corresponding

\footnotetext{
* The author was supported by the Clore scholarship programme and by the Israel MOD Research and Technology Unit.

** The author was supported by the Adams fellowship.

$\star \star \star$ This author was financed by a Ph.D grant of the Katholieke Universiteit Leuven and by the Korea Research Foundation Grant funded by the Korean Government (MOEHRD) (KRF-2005-213-D00077) and supported by the Concerted Research Action (GOA) Ambiorics 2005/11 of the Flemish Government and by the European Commission through the IST Programme under Contract IST2002507932 ECRYPT.
}

E. Biham and A.M. Youssef (Eds.): SAC 2006, LNCS 4356, pp. 2844, 2007.

(C) Springer-Verlag Berlin Heidelberg 2007 
to its number, i.e., SHA-256 has a 256-bit digest, etc. After the publication of these hash functions, NIST has issued another hash function SHA-224 that has a digest size of 224 bits.

Both SHA-0 and SHA-1 were subjected to a great deal of analysis [11. In the last two years there was a major progress in the attacks on both of the hash functions. This progress included finding a collision in SHA-0, and devising an algorithm that can find a collision in SHA-1 in less than $2^{63}$ SHA-1 applications 3430 32 28. The new techniques are based on finding good differentials of the compressing function of SHA-1 and combining them with some novel plaintext modification techniques.

It was suggested to use the compression function of SHA-1 as a block cipher 13. Later this suggestion was named SHACAL-1 and submitted to the NESSIE project [14. SHACAL-1 is a 160-bit block cipher with variable key length (0-512 bits) and 80 rounds based on the compression function of SHA-1. The cipher, was selected as a NESSIE finalist, but was not selected for the NESSIE portfolio [22].

Due to the structure of SHACAL-1, differentials of SHA-1 correspond to related-key differentials of SHACAL-1. Hence, it seems natural that some of the techniques used in the new attacks on SHA-1 can be converted into a relatedkey attack on SHACAL-1. We show that this is indeed the case. The differentials found in the attacks devised in [30] can be converted into high probability related-key differentials of SHACAL-1.

After transforming the collision producing differentials into related-key differentials, we use them in a related-key rectangle attack 8,15,18. The resulting attack succeeds to attack the full 80-round SHACAL-1 using four related-keys faster than exhaustive key search.

The related-key rectangle technique was used in previously published attacks on SHACAL-1 [1518] and was by far the most successful technique to attack the cipher. The best previously known attack on the cipher based on this technique was applicable up to 70 rounds of SHACAL-1. Our results extend these previously known results by using improved differentials and improved attack techniques.

We note that the best known attack on SHACAL-1 that does not use relatedkeys is a rectangle attack on 49-round SHACAL-1 [7]. A comparison of the known attacks along with our new results on SHACAL-1 is presented in Table 1.

This paper is organized as follows: In Section 2 we describe the block cipher SHACAL-1. In Section 3 we describe the previously known results on SHACAL1 and the relevant results on SHA-1. In Section 4 we give a short description of the related-key rectangle attack. In Section 5 we present the new attacks on the full SHACAL-1. Section [6 explores the differences between our attacks on SHACAL-1 and other works on SHA-1. The appendix contains the differentials used in the attack. Finally, Section 7 summarizes the paper.

\section{Description of SHACAL-1}

SHACAL-1 [14] is a 160-bit block cipher supporting variable key lengths (0-512 bits). It is based on the compression function of the hash function SHA-1 [25]. 
Table 1. Summary of Our Results and Previously Known Results on SHACAL-1

\begin{tabular}{|c|c|c|c|c|c|}
\hline \multirow[t]{2}{*}{ Attack \& Source } & \multirow{2}{*}{\multicolumn{2}{|c|}{$\frac{\text { Number of }}{\text { Keys Rounds }}$}} & \multirow{2}{*}{ Rounds } & \multicolumn{2}{|c|}{ Complexity } \\
\hline & & & & Data & Time \\
\hline \begin{tabular}{|l|l} 
Differential 20 \\
\end{tabular} & 1 & 41 & $0-40$ & $2^{141} \mathrm{CP}$ & $2^{491}$ \\
\hline ified Boomerang 20] & 1 & 47 & $0-46$ & $2^{158.5} \mathrm{CP}$ & $2^{508.4}$ \\
\hline gle 7 & 1 & 47 & $0-46$ & $2^{151.9} \mathrm{CP}$ & $2^{482.6}$ \\
\hline Rectangle [7] & 1 & 49 & $29-77$ & $2^{151.9} \mathrm{CC}$ & $2^{508.5}$ \\
\hline Related-Key Rectangle [18 & 2 & 59 & $0-58$ & $2^{149.7} \mathrm{RK}-\mathrm{Cl}$ & $2^{498.3}$ \\
\hline Related-Key Rectangle 15 & 4 & 70 & $0-69$ & $2^{151.8} \mathrm{RK}-\mathrm{Cl}$ & $2^{500.1}$ \\
\hline Related-Key Rectangle (Sec & 4 & $\overline{80}$ & $0-79$ & $2^{159.8} \mathrm{RK}-\mathrm{Cl}$ & $2^{420.0}$ \\
\hline Related-Key Rectangle (Section 5.2 ) & 4 & 80 & $0-79$ & $2^{153.8} \mathrm{RK}-\mathrm{Cl}$ & $2^{501.2}$ \\
\hline
\end{tabular}

Complexity is measured in encryption units.

$\mathrm{CP}$ - Chosen Plaintexts, CC — Chosen Ciphertexts, RK — Related-Key.

The cipher has 80 rounds (also referred as steps) grouped into four types of 20 rounds each 1

The 160-bit plaintext is divided into five 32-bit words - A, B, C, D and $E$. We denote by $X_{i}$ the value of word $X$ before the $i$ th round, i.e., the plaintext $P$ is divided into $A_{0}, B_{0}, C_{0}, D_{0}$ and $E_{0}$, and the ciphertext is composed of $A_{80}, B_{80}, C_{80}, D_{80}$ and $E_{80}$.

In each round the words are updated according to the following rule:

$$
\begin{aligned}
A_{i+1} & =W_{i}+\operatorname{ROTL}_{5}\left(A_{i}\right)+f_{i}\left(B_{i}, C_{i}, D_{i}\right)+E_{i}+K_{i} \\
B_{i+1} & =A_{i} \\
C_{i+1} & =\operatorname{ROTL}_{30}\left(B_{i}\right) \\
D_{i+1} & =C_{i} \\
E_{i+1} & =D_{i}
\end{aligned}
$$

where + denotes addition modulo $2^{32}, \operatorname{ROTL}_{j}(X)$ represents rotation to the left by $j$ bits, $W_{i}$ is the round subkey, and $K_{i}$ is the round constant 2 There are three different functions $f_{i}$, selected according to the round number:

$$
\begin{array}{lc}
f_{i}(X, Y, Z)=f_{i f}=(X \& Y) \mid(\neg X \& Z) & 0 \leq i \leq 19 \\
f_{i}(X, Y, Z)=f_{\text {xor }}=(X \oplus Y \oplus Z) & 20 \leq i \leq 39,60 \leq i \leq 79 \\
f_{i}(X, Y, Z)=f_{\text {maj }}=((X \& Y)|(X \& Z)|(Y \& Z)) & 40 \leq i \leq 59
\end{array}
$$

In [14] it is strongly advised to use keys of at least 128 bits, even though shorter keys are supported. The first step in the key schedule algorithm is to pad the supplied key into a 512-bit key. Then, the 512-bit key is expanded into eighty 32-bit subkeys (or a total of 2560 bits of subkey material). The

\footnotetext{
${ }^{1}$ To avoid confusion, we adopt the common notations for rounds. In [14] the notation step stands for round, where round is used for a group of 20 steps.

${ }^{2}$ This time we adopt the notations of [14], and alert the reader of the somewhat confusing notations.
} 
expansion is done in a linear manner using a linear feedback shift register (over $\left.G F\left(2^{32}\right)\right)$.

The key schedule is as follows: Let $M_{0}, \ldots, M_{15}$ be the 16 key words (32 bits each). Then the round subkeys $W_{0}, \ldots, W_{79}$ are computed by the following algorithm:

$$
W_{i}= \begin{cases}M_{i} & 0 \leq i \leq 15 \\ \left(W_{i-3} \oplus W_{i-8} \oplus W_{i-14} \oplus W_{i-16}\right) \ll 1 & 16 \leq i \leq 79 .\end{cases}
$$

\section{Previous Results}

A preliminary differential and linear analysis of the properties of the compression function of SHA-1 as a block cipher is presented in 13. The found differentials are relatively short (10 rounds) and have probabilities varying between $2^{-13}$ and $2^{-26}$ (depending on the round functions).

In [26] these differentials are improved, and 20-round differentials with probability $2^{-41}$ are presented. In [20] another set of differentials of SHACAL-1 is presented, including a 30-round differential with probability $2^{-130}$.

In 24] an algorithm for identifying whether two SHACAL-1 encryptions use a pair of related keys is presented. The attack is based on finding slid pairs. Once a slid pair is encountered, the attacker can determine whether the two encryptions have related keys. The attack requires about $2^{96}$ encryptions under each of the two keys to find a slid pair.

In 20] a 21-round differential for rounds $0-20$ and a 15-round differential for rounds 21-35 are combined to devise an amplified boomerang distinguisher [16 for 36-round SHACAL-1. This distinguisher is used to attack 39-round SHACAL-1 using $2^{158.5}$ chosen plaintexts and about $2^{250.8} 39$-round SHACAL- 1 encryptions. The attack is based on guessing (or trying) the subkeys of the three additional rounds, and then checking whether the distinguisher succeeds. This approach is further extended to attack 47-round SHACAL-1 before exhaustive key search becomes faster than this attack. Another attack presented in 20] is a differential attack on 41-round SHACAL-1. The success of these attacks was questioned and resolved in [7].

Besides resolving the problems with previous attacks, in [7] a rectangle attack on 49-round SHACAL-1 is presented. The attack requires $2^{151.9}$ chosen plaintexts, and has a running time equivalent to $2^{508.5}$ 49-round SHACAL-1 encryptions.

In [18] a related-key rectangle attack with two keys is presented against 59round SHACAL-1. This attack has a data complexity of $2^{149.7}$ related-key chosen plaintexts and has a time complexity of $2^{498.3} 59$-round SHACAL-1 encryptions. This attack is improved in 15] to a related-key rectangle attack with four keys on 70-round SHACAL-1. The improved attack has a data complexity of $2^{151.8}$ related-key chosen plaintexts, and a time complexity of $2^{500.1} 70$-round SHACAL-1 encryptions. 


\section{Related-Key Boomerang and Related-Key Rectangle Attacks}

In this section we briefly describe the related-key rectangle attack. First, we outline the boomerang and the rectangle attacks and describe related-key differentials. Then, we describe the combination that forms into the related-key rectangle attack.

\subsection{The Rectangle Attack}

The rectangle attack [5] is an improved variant of the amplified boomerang attack [16] that has evolved from the boomerang attack presented in 27. We first describe the boomerang attack, and then show the transformation into amplified boomerang/rectangle attacks.

The main idea behind the boomerang attack is to use two short differentials with high probabilities instead of one long differential with a low probability. We assume that a block cipher $E:\{0,1\}^{n} \times\{0,1\}^{k} \rightarrow\{0,1\}^{n}$ can be described as a cascade $E=E_{1} \circ E_{0}$, such that for $E_{0}$ there exists a differential $\alpha \rightarrow \beta$ with probability $p$, and for $E_{1}$ there exists a differential $\gamma \rightarrow \delta$ with probability $q$.

The distinguisher is based on the following boomerang process:

- Ask for the encryption of a pair of plaintexts $\left(P_{1}, P_{2}\right)$ such that $P_{1} \oplus P_{2}=\alpha$ and denote the corresponding ciphertexts by $\left(C_{1}, C_{2}\right)$.

- Calculate $C_{3}=C_{1} \oplus \delta$ and $C_{4}=C_{2} \oplus \delta$, and ask for the decryption of the pair $\left(C_{3}, C_{4}\right)$. Denote the corresponding plaintexts by $\left(P_{3}, P_{4}\right)$.

- Check whether $P_{3} \oplus P_{4}=\alpha$.

The boomerang attack uses the first characteristic $(\alpha \rightarrow \beta)$ for $E_{0}$ with respect to the pairs $\left(P_{1}, P_{2}\right)$ and $\left(P_{3}, P_{4}\right)$, and uses the second characteristic $(\gamma \rightarrow \delta)$ for $E_{1}$ with respect to the pairs $\left(C_{1}, C_{3}\right)$ and $\left(C_{2}, C_{4}\right)$.

For a random permutation the probability that the last condition is satisfied is $2^{-n}$. For $E$, the probability that the pair $\left(P_{1}, P_{2}\right)$ is a right pair with respect to the first differential $(\alpha \rightarrow \beta)$ is $p$. The probability that both pairs $\left(C_{1}, C_{3}\right)$ and $\left(C_{2}, C_{4}\right)$ are right pairs with respect to the second differential is $q^{2}$. If all these are right pairs, then $E_{1}^{-1}\left(C_{3}\right) \oplus E_{1}^{-1}\left(C_{4}\right)=\beta=E_{0}\left(P_{3}\right) \oplus E_{0}\left(P_{4}\right)$. Thus, with probability $p, P_{3} \oplus P_{4}=\alpha$. The total probability of this quartet of plaintexts and ciphertexts to satisfy the boomerang conditions is $(p q)^{2}$.

The attack can be mounted for all possible $\beta$ 's and $\gamma$ 's simultaneously (as long as $\beta \neq \gamma$ ). Therefore, a right quartet for $E$ is encountered with probability no less than $(\hat{p} \hat{q})^{2}$, where:

$$
\hat{p}=\sqrt{\sum_{\beta} \operatorname{Pr}^{2}[\alpha \rightarrow \beta]}, \quad \text { and } \quad \hat{q}=\sqrt{\sum_{\gamma} \operatorname{Pr}^{2}[\gamma \rightarrow \delta]} .
$$

The complete analysis is given in [27,56]. 
As the boomerang attack requires adaptive chosen plaintexts and ciphertexts, many of the techniques that were developed for using distinguishers in key recovery attacks can not be combined with the boomerang attack. This led to the introduction of chosen plaintext variants of the boomerang attack called the amplified boomerang attack [16] and the rectangle attack [5]. The transformation of the boomerang attack into a chosen plaintext attack is quite standard, and is achieved by birthday-paradox arguments. The key idea behind the transformation is to encrypt many plaintext pairs with input difference $\alpha$, and to look for quartets that conform to the requirements of the boomerang process.

The rectangle (or the amplified boomerang) process is as follows:

- Ask for the encryption of many pairs of plaintexts $(P, P \oplus \alpha)$.

- Search two pairs of plaintexts $\left(P_{1}, P_{2}\right),\left(P_{3}, P_{4}\right)$, and their corresponding ciphertexts $\left(C_{1}, C_{2}\right)$ and $\left(C_{3}, C_{4}\right)$, respectively, satisfying:

- $P_{1} \oplus P_{2}=P_{3} \oplus P_{4}=\alpha$

- $C_{1} \oplus C_{3}=C_{2} \oplus C_{4}=\delta$

Given the same decomposition of $E$ as before, and the same basic differentials, the analysis in [5] shows that out of $N$ plaintext pairs, the number of right quartets is expected to be $N^{2} 2^{-n} \hat{p}^{2} \hat{q}^{2}$. We note, that the main reduction in the probability follows from the fact that unlike the boomerang attack, in the rectangle attack the event $E_{0}\left(P_{1}\right) \oplus E_{0}\left(P_{3}\right)=\gamma$ occurs with probability $2^{-n}$ even when all the differentials hold.

\subsection{Related-Key Differentials}

Related-key differentials [17] were used for cryptanalysis several times in the past. Recall, that a regular differential deals with some plaintext difference $\Delta P$ and a ciphertext difference $\Delta C$ such that

$$
\operatorname{Pr}_{P, K}\left[E_{K}(P) \oplus E_{K}(P \oplus \Delta P)=\Delta C\right]
$$

is high enough (or zero 2]).

A related-key differential is a triplet of a plaintext difference $\Delta P$, a ciphertext difference $\Delta C$, and a key difference $\Delta K$, such that

$$
\operatorname{Pr}_{P, K}\left[E_{K}(P) \oplus E_{K \oplus \Delta K}(P \oplus \Delta P)=\Delta C\right]
$$

is useful (high enough or zero).

\subsection{Related-Key Rectangle Attack}

The related-key rectangle attack was introduced in [18 15], and independently in 8 .

Let us assume that we have a related-key differential $\alpha \rightarrow \beta$ of $E_{0}$ under a key difference $\Delta K_{a b}$ with probability $p$. Assume also that we have another relatedkey differential $\gamma \rightarrow \delta$ for $E_{1}$ under a key difference $\Delta K_{a c}$ with probability $q$.

The related-key rectangle process involves four different unknown (but related) keys - $K_{a}, K_{b}=K_{a} \oplus \Delta K_{a b}, K_{c}=K_{a} \oplus \Delta K_{a c}$, and $K_{d}=K_{a} \oplus \Delta K_{a b} \oplus$ $\Delta K_{a c}$. The attack is performed by the following algorithm: 


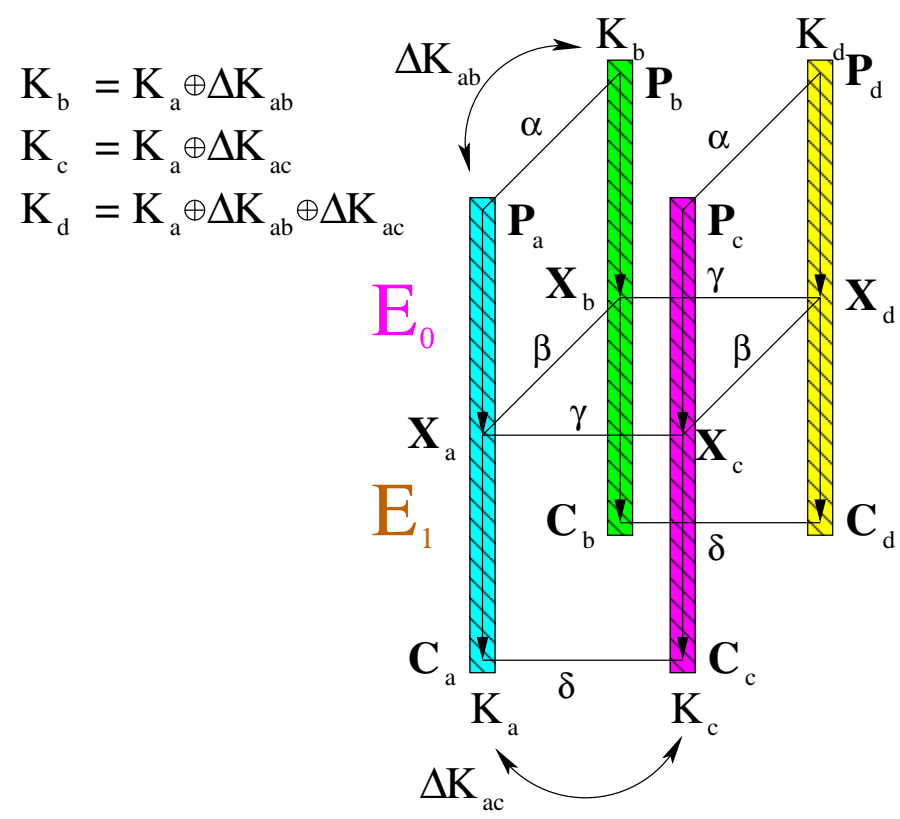

Fig. 1. A Related-Key Rectangle Quartet

- Choose $N$ plaintext pairs $\left(P_{a}, P_{b}=P_{a} \oplus \alpha\right)$ at random and ask for the encryption of $P_{a}$ under $K_{a}$ and of $P_{b}$ under $K_{b}$. Denote the set of these pairs by $S$.

- Choose $N$ plaintext pairs $\left(P_{c}, P_{d}=P_{c} \oplus \alpha\right)$ at random and ask for the encryption of $P_{c}$ under $K_{c}$ and $P_{d}$ under $K_{d}$. Denote the set of these pairs by $T$.

- Search a pair of plaintexts $\left(P_{a}, P_{b}\right) \in S$ and a pair of plaintexts $\left(P_{c}, P_{d}\right) \in$ $T$, and their corresponding ciphertexts $\left(C_{a}, C_{b}\right)$ and $\left(C_{c}, C_{d}\right)$, respectively, satisfying:

- $P_{a} \oplus P_{b}=P_{c} \oplus P_{d}=\alpha$

- $C_{a} \oplus C_{c}=C_{b} \oplus C_{d}=\delta$

See Figure 1 for an outline of such a quartet.

The attack can use many differentials for $E_{0}$ and $E_{1}$ simultaneously (just like in a regular rectangle attack) as long as all related-key differentials used in $E_{0}$ have the same key difference $\Delta K_{a b}$ and the same input difference $\alpha$ and as long as all related-key differentials used in $E_{1}$ have the same key difference $\Delta K_{a c}$ and the same output difference $\delta$.

The analysis of the related-key rectangle attack is similar to the one of the rectangle attack. Starting with $N$ plaintext pairs in $S$ and $N$ plaintext pairs in $T$, we expect to find $N^{2} 2^{-n}(\hat{p} \hat{q})^{2}$ right quartets in $S \times T$. For a random permutation the number of "right quartets" is about $N^{2} 2^{-2 n}$, so as long as $\hat{p} \hat{q}>2^{-n / 2}$ we can use the related-key rectangle attack to distinguish between 
a random permutation and the attacked cipher. This distinguisher can be later used for a key recovery attack.

We note that a related-key boomerang attack can be constructed similarly to the related-key rectangle attack. The full analysis can be found in [8]. The related-key boomerang and rectangle techniques were used to attack reduced round variants of AES, IDEA, SHACAL-1, and SHACAL-2 and the full KASUMI and COCONUT98 8,915,1918].

In the case of SHACAL-1, the key schedule algorithm is linear. Therefore, given a key difference, all subkey differences are known, and can be easily used in the related-key model.

\section{Related-Key Rectangle Attack on the Full SHACAL-1}

Our attack on SHACAL-1 is based on a 69-round related-key distinguisher. In the attack on the full SHACAL-1, we try all the possible subkeys of the remaining 11 rounds, and decrypt all the ciphertexts. Then, the 69-round distinguisher is applied. We improve the time complexity of the attack by partially decrypting only 8 rounds, and then use the early abort approach to reduce the number of values that are decrypted through the remaining three more rounds, before the attack is applied. It is expected that for the right guess of the subkey of the last 11 rounds, the distinguisher would be more successful than for a wrong guess. Thus, we can use this distinguisher to identify (to some extent) the right subkey.

\subsection{9-Round Related-Key Distinguisher}

We decompose 69-round SHACAL-1 into two sub-ciphers: $E_{0}$ that contains the first 34 rounds of SHACAL-1 (rounds 0-33), and $E_{1}$ that contains the remaining 35 rounds (rounds 34-68).

We have transformed the collision producing differentials of SHA-1 presented in 30, into related-key differentials for each of the two sub-ciphers. The first related-key differential (for $E_{0}$ ) has probability $2^{-41}$, and by fixing two bits of the plaintexts and using several differentials simultaneously for $E_{0}$ we obtain $\hat{p}=2^{-38.5}$. The second related-key differential (for $E_{1}$ ) has probability $2^{-39}$, and by using several differentials simultaneously for $E_{1}$ we obtain $\hat{q}=2^{-38.3}$. The differentials are presented in Appendix $\mathrm{A}$.

Combining these two differentials together leads to a 69-round related-key rectangle distinguisher with probability $2^{-80} \cdot \hat{p} \hat{q}=2^{-156.8}$, i.e., given $N$ relatedkey chosen plaintext pairs, we expect $N^{2} \cdot 2^{-160} \cdot(\hat{p} \hat{q})^{2}$ right quartets. Hence, given $2^{157.8}$ related-key chosen plaintext pairs, we expect four right rectangle quartets, while for a random cipher only $2^{-4.4}$ are expected.

\subsection{The Key Recovery Attack}

The basic approach for a key recovery attack is to guess the subkey of the last 11 rounds, partially decrypt all ciphertexts, and apply the distinguisher for the 
remaining 69 rounds. Such an approach can be improved using the fact that in every round, only a small part of the intermediate value is substantially changed, while most of the value is only shifted. The attack is based on the early abort technique which is widely used [1112. In this technique, once a pair/quartet does not satisfy the required differences/properties it is excluded from further analysis.

In the description of the attack algorithm we use the following notations: $X_{A}$ denotes the value of word $A$ in $X$. Similarly, $Y_{D, E}$ denotes words $D$ and $E$ of $Y$, etc. Let $\Delta X_{i}$ denote the difference in word $X$ before round $i$, i.e., $\Delta A_{70}$ is the difference in word $A$ before round 70, and after round 69 . Also, let $e_{i}$ be the 32-bit word composed of 31 0's and 1 in the $i$ th place. We use $e_{i, j}$ to denote $e_{i} \oplus e_{j}$ and $e_{i, j, k}=e_{i, j} \oplus e_{k}$, etc. We also denote the set of possible values of $\Delta A_{70}$ given that the second differential is satisfied by $S^{\prime}$.

We observe that even if we partially decrypt only 8 rounds, we still have a filtering condition on the quartets: Since $\Delta D_{72}=R O T L_{30}\left(\Delta A_{69}\right)$ and $\Delta E_{72}=$ $R O T L_{30}\left(\Delta B_{69}\right)$, we can check whether the difference in these words corresponds to the output difference in words $A$ and $B$ of the second differential. In addition, we observe that we can extend the second differential by a truncated differential of one additional round. There are only $324=2^{8.3}$ possible $\Delta A_{70}$ values in $S^{\prime}$, hence, there are only 324 possible values for $\Delta C_{72}$ in case the second differential holds.

Using these observations, we can get a filtering of $64+23.7=87.7$ bits for every pair in the end of round 71 , or a filtering of 175.4 bits in total. Since the attack starts with $2^{315.6}$ quartets, we expect that $2^{140.3}$ quartets pass the filtering for any given subkey guess of rounds $72-79$. We then guess the subkey of round 71 and compute $\Delta E_{71}$ that is equal to $\Delta C_{69}$ if the differential holds to obtain an additional 64-bit filtering on the remaining quartets. After this filtering only $2^{76.3}$ quartets remain for each subkey guess. Then we continue by guessing the subkeys of rounds 70 and 69 . As a result, the time complexity of the attack drops rapidly, while the data complexity remains unchanged.

The algorithm of the attack is as follows:

\section{Data Collection Phase}

(a) Ask for the encryption of $2^{157.8}$ pairs of plaintexts $\left(P_{a}, P_{b}\right)$, where $P_{b}=$ $P_{a} \oplus \alpha$, where $P_{a}$ and $P_{b}$ satisfy the restrictions described in Appendix $\mathrm{A}$ and where $P_{a}$ is encrypted under $K_{a}$ and $P_{b}$ is encrypted under $K_{b}$.

(b) Ask for the encryption of $2^{157.8}$ pairs of plaintexts $\left(P_{c}, P_{d}\right)$, where $P_{c}=$ $P_{d} \oplus \alpha$, where $P_{c}$ and $P_{d}$ satisfy the restrictions described in Appendix A and where $P_{c}$ is encrypted under $K_{c}$ and $P_{d}$ is encrypted under $K_{d}$.

\section{Partial Decryption}

(a) For each guess of the subkey of rounds 72-79:

i. Partially decrypt all ciphertexts (under the corresponding keys).

ii. Find all pairs of partially decrypted ciphertexts $\left(C_{a}, C_{c}\right)$, such that $C_{a_{C, D}, E} \oplus C_{c_{C, D, E}} \in S$, where $C_{a}$ is encrypted under $K_{a}, C_{c}$ 
is encrypted under $K_{c}$ and $S=\left\{(x, y, z): R O T L_{30}(x) \in S^{\prime}\right.$, $\left.\operatorname{ROTL}_{30}(y)=\delta_{A}=0, \operatorname{ROTL}_{30}(z)=\delta_{B}=e_{2}\right\}$.

iii. For each such pair $\left(C_{a}, C_{c}\right)$, let $P_{a}$ and $P_{c}$ be the corresponding plaintexts. Let $P_{b}=P_{a} \oplus \alpha$ and $P_{d}=P_{c} \oplus \alpha$, and let $C_{b}$ and $C_{d}$ be the corresponding ciphertexts, respectively.

iv. If $C_{b_{C, D}, E} \oplus C_{d_{C, D, E}} \in S$ pass the quartet $\left(P_{a}, P_{b}, P_{c}, P_{d}\right)$ for a further analysis.

(b) Partial Decryption of Round 71: For each guess of the subkey of round 71:

i. Partially decrypt all the remaining quartets (under the corresponding keys) and denote the resulting intermediate values by $\left(C_{a}^{\prime}, C_{b}^{\prime}\right.$, $\left.C_{c}^{\prime}, C_{d}^{\prime}\right)$.

ii. For each of the remaining quartets, check whether $C_{a_{E}}^{\prime} \oplus C_{c_{E}}^{\prime}=\delta_{C}=$ 0 and discard all the quartets that do not satisfy the equation.

iii. For each of the remaining quartets, check whether $C_{b_{E}}^{\prime} \oplus C_{d_{E}}^{\prime}=\delta_{C}=$ 0 and discard all the quartets that do not satisfy the equation.

(c) Partial Decryption of Round 70: For each guess of the subkey of round 70:

i. Partially decrypt all the remaining quartets (under the corresponding keys) and denote the resulting intermediate values by $\left(C_{a}^{\prime \prime}, C_{b}^{\prime \prime}\right.$, $\left.C_{c}^{\prime \prime}, C_{d}^{\prime \prime}\right)$.

ii. For each of the remaining quartets, check whether $C_{a_{E}}^{\prime \prime} \oplus C_{c_{E}}^{\prime \prime}=\delta_{D}=$ 0 and discard all the quartets that do not satisfy the equation.

iii. For each of the remaining quartets, check whether $C_{b_{E}}^{\prime} \oplus C_{d_{E}}^{\prime}=\delta_{D}=$ 0 and discard all the quartets that do not satisfy the equation.

(d) Partial Decryption of Round 69: For each guess of the subkey of round 69:

i. Partially decrypt all the remaining quartets (under the corresponding keys) and denote the resulting intermediate values by $\left(C_{a}^{\prime \prime \prime}, C_{b}^{\prime \prime \prime}\right.$, $\left.C_{c}^{\prime \prime \prime}, C_{d}^{\prime \prime \prime}\right)$.

ii. For each of the remaining quartets, check whether $C_{a_{E}}^{\prime \prime \prime} \oplus C_{c_{E}}^{\prime \prime \prime}=\delta_{E}=$ $e_{1}$ and discard all the quartets that do not satisfy the equation.

iii. For each of the remaining quartets, check whether $C_{b_{E}}^{\prime \prime \prime} \oplus C_{d_{E}}^{\prime \prime \prime}=\delta_{E}=$ $e_{1}$ and discard all the quartets that do not satisfy the equation.

iv. Pass all the remaining quartets to further analysis.

(e) Further Analysis: If for this subkey guess only one quartet is suggested (or no quartets are suggested) discard the subkey guess. If the subkey is not discarded, exhaustively search all possible values for the remaining 160 subkey bits for the correct key.

\subsection{Analysis of the Key Recovery Attack}

The time complexity of Step 1 is $2^{159.8}$ encryptions. The time complexity of Step $2(\mathrm{a})$ is $\frac{8}{80} \cdot 2^{256} \cdot 2^{159.8}=2^{412.5}$ SHACAL-1 encryptions. Steps $2(\mathrm{~b})-2(\mathrm{e})$ 
are repeated for each subkey guess, i.e., $2^{256}$ times. For a given subkey guess, Step 2(b) consists of $2^{141.3} \cdot 2^{32}$ partial decryptions of one SHACAL-1 round. This is equivalent to $2^{141.3} \cdot 2^{32} \cdot \frac{1}{80}=2^{167.0}$ full SHACAL- 1 encryptions. Thus, the total time complexity of Step 2 (b) is about $2^{256} \cdot 2^{167.0}=2^{423.0}$ SHACAL-1 encryptions.

There is an improvement of the time complexity by a factor of 8 based on the observation that the difference in the most significant bit is not affected by the actual key value. Thus, it is possible to guess in Step 2(a) the entire subkey of rounds $74-79$, and all but most significant bits of the subkeys of rounds $72-73$. This does not affect the ability to compute the difference in the most significant bits of the words $D^{72}$ and $E^{72}$. Similarly, in Step 2(b) is is sufficient to guess the 31 least significant bits of $K_{71}$ in order to find the difference in the three words: $C^{71}, D^{71}$, and $E^{71}$.

In Step 2(c) there is again no need to guess the entire subkey to deduce the difference in the most significant bit. However, in order for the partial decryption to be done correctly, the real value of the $B^{70}$ has to be computed. Thus, in this step we guess the most significant bit of $K^{73}$ along with the 31 least significant bits of $K^{70}$. The same is done also in Step 2(d), where the most significant bit of $K^{72}$ is guessed along with the 31 least significant bits of $K^{69}$. We note that the improved variant guess $11 \cdot 32-3=349$ subkey bits during the entire attack.

The time complexities of the other steps are relatively smaller. Hence, the total data complexity of the attack is $2^{159.8}$ related-key chosen plaintexts encrypted under four keys, and the time complexity is $2^{420.0}$ SHACAL- 1 encryptions. The memory requirement of the attack is about $2^{159.8}$ memory blocks of 320 bits, required for storing the large amount of data.

We note that a different approach may be used in our attack. We can remove the last three rounds of the second differential to increase its probability by a factor of $2^{6}$, resulting in a 66-round related-key rectangle distinguisher with probability $2^{-80} \cdot \hat{p} \cdot \hat{q}=2^{-150.8}$. The resultant distinguisher requires $2^{151.8}$ related-key chosen plaintext pairs $\left(P_{a}, P_{b}\right)$ and $\left(P_{c}, P_{d}\right)$ each to produce four right plaintext quartets (while for a random cipher about $2^{-16.4}$ quartets that satisfy the rectangle conditions are expected). Then, we apply partial decryptions of rounds 69-79, 68, 67 and 66 in Steps 2(a), 2(b), 2(c) and 2(d), respectively, and then run the final exhaustive search for the remaining 64-bit keys in Step 2(e).

The time complexity of Step $2(\mathrm{a})$ in this case is $2^{152.8+352} \cdot(11 / 80)=2^{501.9}$ SHACAL-1 encryptions. In this attack we can derive the set $S$ in Step 2-(a) for the filtering of quartets, which has $2^{70.8}$ elements, and thus the number of remaining quartets after this step is about $\left(2^{151.9} \cdot 2^{-160+70.8}\right)^{2}=2^{125.2}$. It follows that Step 2 (b) takes about $2^{126.2} \cdot 2^{352+32} \cdot(1 / 80)=2^{503.9}$ SHACAL-1 encryptions. Compared to Steps 2(a) and 2(b), the followed steps have quite small time complexities. Hence, this full-round attack on SHACAL-1 works with a data complexity of $2^{153.8}$ related-key chosen plaintexts encrypted under four related keys and with a time complexity of $2^{501.9}+2^{503.9}=2^{504.2}$ SHACAL- 1 
encryptions. Again, a factor 8 in the time complexity can be improved using the observation about the most significant bits, i.e., the attack's time complexity is $2^{501.2}$ SHACAL-1 encryptions.

\section{Differences Between Attacking SHA-1 and SHACAL-1}

While it may seem that any attack on SHA-1 can be easily transformed into an attack on SHACAL-1, and vice versa, this is not exactly the case. Investigating the recent attacks on SHA-1 in 3430, it seems that these attacks heavily rely on the fact that the attacker can control some of the bits that enter the nonlinear operations. This way, the collision-producing differentials have much higher probability than the respective related-key differentials we use. We can impose conditions on the keys (increasing the probabilities of the related-key differentials), but then our attack would be applicable only for such keys, i.e., a weak key class.

Another difference between the attacks on SHA-1 and our attack is the fact that the collision attacks can iteratively fix the values they use, i.e., using message modification techniques or neutral bits. This enables the collision producing attacks to use shorter differential than ours (as these attacks actually start the probabilistic process in a much later step).

There is another difference between the two cases. While in the case of encryption (SHACAL-1), we have to deal with each block of message independently, collision attacks on the hash function can use multiple blocks. For example, the attacker can treat messages that detoured the differential in a very late step, by respective changes to the second block of the message. This fact allows the collision search to use shorter differentials (this time from the end point), thus, increasing the success probability.

Another problem our attack faces is the dual representation of XOR and additive differentials. As we have less control on the encryption process than the collision attacks have on the compression process, it is less useful for us to consider the differentials using the dual representation. Again, for exploiting the advantage of the additive differentials in the related-key differentials, we must fix some of the key bits, resulting again in a weak key class.

\section{Summary and Conclusions}

In this paper we converted the differentials of the compression function of SHA-1 presented by Wang et al. to related-key differentials of the block cipher SHACAL1. Then we used the related-key rectangle technique to devise the first known attack on the full 80-round SHACAL-1.

We also discussed the possibility of converting other techniques used in the attacks on SHA-1 to attack SHACAL-1, and concluded that such conversion will result in an attack applicable only to a weak key class of SHACAL-1. 
Our attack improves by far the previously known results, that were able to attack up to 70 rounds of the cipher, and demonstrates the power of the related-key rectangle technique. However, the result is still highly theoretical and a practical attack on the full SHACAL-1 seems out of reach at this stage. We note that keys shorter than 420 bits can still be considered secure, as for these keys the time complexity of our attack is greater than exhaustive key search.

\section{References}

1. Biham, E.: New Types of Cryptanalytic Attacks Using Related Keys. Journal of Cryptology 7(4), 229-246 (1994)

2. Biham, E., Biryukov, A., Shamir, A.: Cryptanalysis of Skipjack Reduced to 31 Rounds Using Impossible Differentials. In: Stern, J. (ed.) EUROCRYPT 1999. LNCS, vol. 1592, pp. 12-23. Springer, Heidelberg (1999)

3. Biham, E., Chen, R.: Near-Collisions of SHA-0. In: Franklin, M. (ed.) CRYPTO 2004. LNCS, vol. 3152, pp. 290-305. Springer, Heidelberg (2004)

4. Biham, E., Chen, R., Joux, A., Carribault, P., Lemuet, C., Jalby, W.: Collisions of SHA-0 and Reduced SHA-1. In: Cramer, R.J.F. (ed.) EUROCRYPT 2005. LNCS, vol. 3494, pp. 36-57. Springer, Heidelberg (2005)

5. Biham, E., Dunkelman, O., Keller, N.: The Rectangle Attack - Rectangling the Serpent. In: Pfitzmann, B. (ed.) EUROCRYPT 2001. LNCS, vol. 2045, pp. 340 357. Springer, Heidelberg (2001)

6. Biham, E., Dunkelman, O., Keller, N.: New Results on Boomerang and Rectangle Attacks. In: Daemen, J., Rijmen, V. (eds.) FSE 2002. LNCS, vol. 2365, pp. 1-16. Springer, Heidelberg (2002)

7. Biham, E., Dunkelman, O., Keller, N.: Rectangle Attacks on 49-Round SHACAL-1. In: Johansson, T. (ed.) FSE 2003. LNCS, vol. 2887, pp. 22-35. Springer, Heidelberg (2003)

8. Biham, E., Dunkelman, O., Keller, N.: Related-Key Boomerang and Rectangle Attacks. In: Cramer, R.J.F. (ed.) EUROCRYPT 2005. LNCS, vol. 3494, pp. $507-$ 525. Springer, Heidelberg (2005)

9. Biham, E., Dunkelman, O., Keller, N.: A Related-Key Rectangle Attack on the Full KASUMI. In: Roy, B. (ed.) ASIACRYPT 2005. LNCS, vol. 3788, pp. 443-461. Springer, Heidelberg (2005)

10. Biham, E., Shamir, A.: Differential Cryptanalysis of the Data Encryption Standard. Springer, Heidelberg (1993)

11. Chabaud, F., Joux, A.: Differential Collisions in SHA-0. In: Krawczyk, H. (ed.) CRYPTO 1998. LNCS, vol. 1462, pp. 56-71. Springer, Heidelberg (1998)

12. Ferguson, N., Kelsey, J., Lucks, S., Schneier, B., Stay, M., Wagner, D., Whiting, D.: Improved Cryptanalysis of Rijndael. In: Schneier, B. (ed.) FSE 2000. LNCS, vol. 1978, pp. 213-230. Springer, Heidelberg (2001)

13. Handschuh, H., Knudsen, L.R., Robshaw, M.J.: Analysis of SHA-1 in Encryption Mode. In: Naccache, D. (ed.) CT-RSA 2001. LNCS, vol. 2020, pp. 70-83. Springer, Heidelberg (2001)

14. Handschuh, H., Naccache, D.: SHACAL. In: preproceedings of NESSIE first workshop, Leuven (2000)

15. Hong, S., Kim, J., Kim, G., Lee, S., Preneel, B.: Related-Key Rectangle Attacks on Reduced Versions of SHACAL-1 and AES-192. In: Gilbert, H., Handschuh, H. (eds.) FSE 2005. LNCS, vol. 3557, pp. 368-383. Springer, Heidelberg (2005) 
16. Kelsey, J., Kohno, T., Schneier, B.: Amplified Boomerang Attacks Against Reduced-Round MARS and Serpent. In: Schneier, B. (ed.) FSE 2000. LNCS, vol. 1978, pp. 75-93. Springer, Heidelberg (2001)

17. Kelsey, J., Schneier, B., Wagner, D.: Key-Schedule Cryptoanalysis of IDEA, GDES, GOST, SAFER, and Triple-DES. In: Koblitz, N. (ed.) CRYPTO 1996. LNCS, vol. 1109, pp. 237-251. Springer, Heidelberg (1996)

18. Kim, J., Kim, G., Hong, S., Lee, S., Hong, D.: The Related-Key Rectangle Attack - Application to SHACAL-1. In: Wang, H., Pieprzyk, J., Varadharajan, V. (eds.) ACISP 2004. LNCS, vol. 3108, pp. 123-136. Springer, Heidelberg (2004)

19. Kim, J., Kim, G., Lee, S., Lim, J., Song, J.: Related-Key Attacks on Reduced Rounds of SHACAL-2. In: Canteaut, A., Viswanathan, K. (eds.) INDOCRYPT 2004. LNCS, vol. 3348, pp. 175-189. Springer, Heidelberg (2004)

20. Kim, J., Moon, D., Lee, W., Hong, S., Lee, S., Jung, S.: Amplified Boomerang Attack against Reduced-Round SHACAL. In: Zheng, Y. (ed.) ASIACRYPT 2002. LNCS, vol. 2501, pp. 243-253. Springer, Heidelberg (2002)

21. NESSIE - New European Schemes for Signatures, Integrity and Encryption, http://www.nessie.eu.org/nessie

22. NESSIE, Portfolio of recommended cryptographic primitives

23. NESSIE, Performance of Optimized Implementations of the NESSIE Primitives, NES/DOC/TEC/WP6/D21/2

24. Saarinen, M.-J.O.: Cryptanalysis of Block Ciphers Based on SHA-1 and MD5. In: Johansson, T. (ed.) FSE 2003. LNCS, vol. 2887, pp. 36-44. Springer, Heidelberg (2003)

25. National, U.S.: Bureau of Standards, Secure Hash Standard, Federal Information Processing Standards Publications No. 180-2 (2002)

26. Bogeart, E.V.D., Rijmen, V.: Differential Analysis of SHACAL, NESSIE internal report NES/DOC/KUL/WP3/009/a (2001)

27. Wagner, D.: The Boomerang Attack. In: Knudsen, L.R. (ed.) FSE 1999. LNCS, vol. 1636, pp. 156-170. Springer, Heidelberg (1999)

28. Wang, X., Yao, A.C., Yao, F.: Cryptanalysis on SHA-1. In: Cryptographic Hash Workshop, NIST, Gaithersburg (2005)

29. Wang, X., Lai, X., Feng, D., Chen, H., Yu, X.: Cryptanalysis of the Hash Functions MD4 and RIPEMD. In: Cramer, R.J.F. (ed.) EUROCRYPT 2005. LNCS, vol. 3494, pp. 1-18. Springer, Heidelberg (2005)

30. Wang, X., Yin, Y.L., Yu, H.: Finding Collisions in the Full SHA-1. In: Shoup, V. (ed.) CRYPTO 2005. LNCS, vol. 3621, pp. 17-36. Springer, Heidelberg (2005)

31. Wang, X., Yu, H.: How to Break MD5 and Other Hash Functions. In: Cramer, R.J.F. (ed.) EUROCRYPT 2005. LNCS, vol. 3494, pp. 19-35. Springer, Heidelberg (2005)

32. Wang, X., Yu, H., Yin, Y.L.: Efficient Collision Search Attacks on SHA-0. In: Shoup, V. (ed.) CRYPTO 2005. LNCS, vol. 3621, pp. 1-16. Springer, Heidelberg (2005)

\section{A Related-Key Differentials of SHACAL-1}

In this appendix we describe the differentials used for our related-key rectangle attacks on SHACAL-1. These differentials are based on the collision producing differentials presented in 30 . 
Table 2. Related-Key Differential for Rounds 0-33 of SHACAL-1

\begin{tabular}{|c|c|c|c|c|c|c|c|}
\hline Round (i) & $\Delta K$ & $\Delta A_{i}$ & $\Delta B_{i}$ & $\Delta C_{i}$ & $\Delta D_{i}$ & $\Delta E_{i}$ & Probability \\
\hline Input 0 & $e_{1}$ & 0 & 0 & $e_{31}$ & $e_{31}$ & $e_{31}$ & $2^{-1}$ \\
\hline $1^{\dagger}$ & $e_{6}$ & $e_{1}$ & 0 & 0 & $e_{31}$ & $e_{31}$ & $2^{-2}$ \\
\hline $2^{\dagger}$ & $e_{1,31}$ & 0 & $e_{1}$ & 0 & 0 & $e_{31}$ & $2^{-2}$ \\
\hline 3 & $e_{31}$ & 0 & 0 & $e_{31}$ & 0 & 0 & $2^{-1}$ \\
\hline 4 & $e_{1,31}$ & 0 & 0 & 0 & $e_{31}$ & 0 & $2^{-2}$ \\
\hline 5 & $e_{6,31}$ & $e_{1}$ & 0 & 0 & 0 & $e_{31}$ & $2^{-1}$ \\
\hline 6 & 0 & 0 & $e_{1}$ & 0 & 0 & 0 & $2^{-2}$ \\
\hline 7 & $e_{6,31}$ & $e_{1}$ & 0 & $e_{31}$ & 0 & 0 & $2^{-2}$ \\
\hline 8 & $e_{31}$ & 0 & $e_{1}$ & 0 & $e_{31}$ & 0 & $2^{-3}$ \\
\hline 9 & $e_{6}$ & $e_{1}$ & 0 & $e_{31}$ & 0 & $e_{31}$ & $2^{-2}$ \\
\hline 10 & $e_{31}$ & 0 & $e_{1}$ & 0 & $e_{31}$ & 0 & $2^{-3}$ \\
\hline 11 & $e_{6}$ & $e_{1}$ & 0 & $e_{31}$ & 0 & $e_{31}$ & $2^{-2}$ \\
\hline 12 & $e_{1,31}$ & 0 & $e_{1}$ & 0 & $e_{31}$ & 0 & $2^{-3}$ \\
\hline 13 & 0 & 0 & 0 & $e_{31}$ & 0 & $e_{31}$ & $2^{-1}$ \\
\hline 14 & $e_{31}$ & 0 & 0 & 0 & $e_{31}$ & 0 & $2^{-1}$ \\
\hline 15 & $e_{31}$ & 0 & 0 & 0 & 0 & $e_{31}$ & 1 \\
\hline 16 & 0 & 0 & 0 & 0 & 0 & 0 & 1 \\
\hline 17 & 0 & 0 & 0 & 0 & 0 & 0 & 1 \\
\hline 18 & 0 & 0 & 0 & 0 & 0 & 0 & 1 \\
\hline 19 & 0 & 0 & 0 & 0 & 0 & 0 & 1 \\
\hline 20 & 0 & 0 & 0 & 0 & 0 & 0 & 1 \\
\hline 21 & 0 & 0 & 0 & 0 & 0 & 0 & 1 \\
\hline 22 & 0 & 0 & 0 & 0 & 0 & 0 & 1 \\
\hline 23 & 0 & 0 & 0 & 0 & 0 & 0 & 1 \\
\hline 24 & 0 & 0 & 0 & 0 & 0 & 0 & 1 \\
\hline 25 & 0 & 0 & 0 & 0 & 0 & 0 & 1 \\
\hline 26 & $e_{2}$ & 0 & 0 & 0 & 0 & 0 & $2^{-1}$ \\
\hline 27 & $e_{7}$ & $e_{2}$ & 0 & 0 & 0 & 0 & $2^{-1}$ \\
\hline 28 & $e_{2}$ & 0 & $e_{2}$ & 0 & 0 & 0 & $2^{-1}$ \\
\hline 29 & $e_{0,3}$ & 0 & 0 & $e_{0}$ & 0 & 0 & $2^{-2}$ \\
\hline 30 & $e_{0,8}$ & $e_{3}$ & 0 & 0 & $e_{0}$ & 0 & $2^{-2}$ \\
\hline 31 & $e_{0,3}$ & 0 & $e_{3}$ & 0 & 0 & $e_{0}$ & $2^{-2}$ \\
\hline 32 & $e_{1,4}$ & 0 & 0 & $e_{1}$ & 0 & 0 & $2^{-2}$ \\
\hline 33 & $e_{1,9}$ & $e_{4}$ & 0 & 0 & $e_{1}$ & 0 & $2^{-2}$ \\
\hline Output (34) & & 0 & $e_{4}$ & 0 & 0 & $e_{1}$ & \\
\hline
\end{tabular}

${ }^{\top}$ - The probability of this round can be improved by a factor of 2 .

Differences are presented before the round, i.e., $\Delta A_{0}$ is the input difference.

The first related-key differential is for rounds 0-33 and is presented in Table 2 , The probability of the differential is $2^{-41}$. This probability can be increased by a factor of 4 by fixing the equivalent to two bits in each of the plaintexts of the pair. If we set the most significant bit of $A$ to be zero, the probability of the second round of the differential is increased by a factor of 2 . By setting bit 3 of $A$ to differ from bit 3 of $B$, the probability of the third round of the differential is also increased by a factor of 2 . 
Table 3. Related-Key Differential for Rounds 34-68 of SHACAL-1

\begin{tabular}{|c|c|c|c|c|c|c|c|}
\hline Round (i) & $\Delta K$ & $\Delta A_{i}$ & $\Delta B_{i}$ & $\overline{\Delta C_{i}}$ & $\overline{\Delta D_{i}}$ & $\Delta E_{i}$ & Probability \\
\hline Input 34 & $\overline{e_{1,30}}$ & 0 & $e_{1}$ & $e_{31}$ & 0 & $e_{30,31}$ & $2^{-2}$ \\
\hline 35 & $e_{1}$ & 0 & 0 & $e_{31}$ & $e_{31}$ & 0 & $2^{-1}$ \\
\hline 36 & $e_{6}$ & $e_{1}$ & 0 & 0 & $e_{31}$ & $e_{31}$ & $2^{-1}$ \\
\hline 37 & $e_{1,31}$ & 0 & $e_{1}$ & 0 & 0 & $e_{31}$ & $2^{-1}$ \\
\hline 38 & $e_{31}$ & 0 & 0 & $e_{31}$ & 0 & 0 & 1 \\
\hline 39 & $e_{1,31}$ & 0 & 0 & 0 & $e_{31}$ & 0 & $2^{-1}$ \\
\hline 40 & $e_{6,31}$ & $e_{1}$ & 0 & 0 & 0 & $e_{31}$ & $2^{-1}$ \\
\hline 41 & 0 & 0 & $e_{1}$ & 0 & 0 & 0 & $2^{-2}$ \\
\hline 42 & $e_{6,31}$ & $e_{1}$ & 0 & $e_{31}$ & 0 & 0 & $2^{-2}$ \\
\hline 43 & $e_{31}$ & 0 & $e_{1}$ & 0 & $e_{31}$ & 0 & $2^{-3}$ \\
\hline 44 & $e_{6}$ & $e_{1}$ & 0 & $e_{31}$ & 0 & $e_{31}$ & $2^{-2}$ \\
\hline 45 & $e_{31}$ & 0 & $e_{1}$ & 0 & $e_{31}$ & 0 & $2^{-3}$ \\
\hline 46 & $e_{6}$ & $e_{1}$ & 0 & $e_{31}$ & 0 & $e_{31}$ & $2^{-2}$ \\
\hline 47 & $e_{1,31}$ & 0 & $e_{1}$ & 0 & $e_{31}$ & 0 & $2^{-3}$ \\
\hline 48 & 0 & 0 & 0 & $e_{31}$ & 0 & $e_{31}$ & $2^{-1}$ \\
\hline 49 & $e_{31}$ & 0 & 0 & 0 & $e_{31}$ & 0 & $2^{-1}$ \\
\hline 50 & $e_{31}$ & 0 & 0 & 0 & 0 & $e_{31}$ & 1 \\
\hline 51 & 0 & 0 & 0 & 0 & 0 & 0 & 1 \\
\hline 52 & 0 & 0 & 0 & 0 & 0 & 0 & 1 \\
\hline 53 & 0 & 0 & 0 & 0 & 0 & 0 & 1 \\
\hline 54 & 0 & 0 & 0 & 0 & 0 & 0 & 1 \\
\hline 55 & 0 & 0 & 0 & 0 & 0 & 0 & 1 \\
\hline 56 & 0 & 0 & 0 & 0 & 0 & 0 & 1 \\
\hline 57 & 0 & 0 & 0 & 0 & 0 & 0 & 1 \\
\hline 58 & 0 & 0 & 0 & 0 & 0 & 0 & 1 \\
\hline 59 & 0 & 0 & 0 & 0 & 0 & 0 & 1 \\
\hline 60 & 0 & 0 & 0 & 0 & 0 & 0 & 1 \\
\hline 61 & $e_{2}$ & 0 & 0 & 0 & 0 & 0 & $2^{-1}$ \\
\hline 62 & $e_{7}$ & $e_{2}$ & 0 & 0 & 0 & 0 & $2^{-1}$ \\
\hline 63 & $e_{2}$ & 0 & $e_{2}$ & 0 & 0 & 0 & $2^{-1}$ \\
\hline 64 & $e_{0,3}$ & 0 & 0 & $e_{0}$ & 0 & 0 & $2^{-2}$ \\
\hline 65 & $e_{0,8}$ & $e_{3}$ & 0 & 0 & $e_{0}$ & 0 & $2^{-2}$ \\
\hline 66 & $e_{0,3}$ & 0 & $e_{3}$ & 0 & 0 & $e_{0}$ & $2^{-2}$ \\
\hline 67 & $e_{1,4}$ & 0 & 0 & $e_{1}$ & 0 & 0 & $2^{-2}$ \\
\hline 68 & $e_{1,9}$ & $e_{4}$ & 0 & 0 & $e_{1}$ & 0 & $2^{-2}$ \\
\hline Output 69 & & 0 & $e_{4}$ & 0 & 0 & $e_{1}$ & \\
\hline
\end{tabular}

Differences are presented before the round, i.e., $\Delta A_{34}$ is the input difference.

We use the notation $e_{i}$ to represent the 32-bit word composed of 310 's and 1 in the $i$ th place. We use $e_{i, j}$ to denote $e_{i} \oplus e_{j}$ and $e_{i, j, k}=e_{i, j} \oplus e_{k}$, etc.

Due to the nature of the rectangle attack, we can improve the probability by counting over several differentials. We have counted over differentials which have the same first 33 rounds as the differential presented in Table 2. The resulting probability is $\hat{p}=2^{-38.5}$ (when fixing the respective bits of the plaintext). 
The second related-key differential for rounds $34-68$ is presented in Table 3. This differential is also based on the collision producing differentials of [30]. The probability of this differential is $2^{-39}$.

Again, due to the nature of the rectangle attack, we can improve the probability by counting over several differentials. We count over various similar characteristics, by changing the first round of this differential. The resulting probability is $\hat{q}=2^{-38.3}$. 